\title{
Pharmacological inhibition of Akt and downstream pathways modulates the expression of COX-2 and mPGES-1 in activated microglia
}

\author{
Antonio CP de Oliveira ${ }^{1,2}$, Eduardo Candelario-Jalil ${ }^{1,3}$, Julia Langbein ${ }^{1}$, Lena Wendeburg ${ }^{1}$, Harsharan S Bhatia',
} Johannes CM Schlachetzki ${ }^{1,4}$, Knut Biber ${ }^{1}$ and Bernd L Fiebich ${ }^{1,5^{*}}$

\begin{abstract}
Background: Microglia are considered a major target for modulating neuroinflammatory and neurodegenerative disease processes. Upon activation, microglia secrete inflammatory mediators that contribute to the resolution or to further enhancement of damage in the central nervous system (CNS). Therefore, it is important to study the intracellular pathways that are involved in the expression of the inflammatory mediators. Particularly, the role of the phosphatidylinositol 3-kinase (PI3K)/Akt/mammalian target of rapamycin (mTOR) and glycogen synthase kinase-3 (GSK-3) pathways in activated microglia is unclear. Thus, in the present study we investigated the role of Akt and its downstream pathways, GSK-3 and mTOR, in lipopolysaccharide (LPS)-activated primary rat microglia by pharmacological inhibition of these pathways in regard to the expression of cyclooxygenase (COX)-2 and microsomal prostaglandin E synthase-1 (mPGES-1) and to the production of prostaglandin (PG) $E_{2}$ and $\mathrm{PGD}_{2}$.

Findings: We show that inhibition of Akt by the Akt inhibitor $X$ enhanced the production of $\mathrm{PGE}_{2}$ and $P G D_{2}$ without affecting the expression of COX-2, mPGES-1, mPGES-2 and cytosolic prostaglandin E synthase (cPGES). Moreover, inhibition of GSK-3 reduced the expression of both COX-2 and MPGES-1. In contrast, the mTOR inhibitor rapamycin enhanced both COX-2 and mPGES-1 immunoreactivity and the release of $\mathrm{PGE}_{2}$ and $\mathrm{PGD}_{2}$. Interestingly, NVP-BEZ235, a dual PI3K/mTOR inhibitor, enhanced COX-2 and reduced mPGES-1 immunoreactivity, albeit PGE 2 and $P G D_{2}$ levels were enhanced in LPS-stimulated microglia. However, this compound also increased $P G E_{2}$ in nonstimulated microglia.

Conclusion: Taken together, we demonstrate that blockade of mTOR and/or PI3K/Akt enhances prostanoid production and that PI3K/Akt, GSK-3 and mTOR differently regulate the expression of mPGES-1 and COX-2 in activated primary microglia. Therefore, these pathways are potential targets for the development of novel strategies to modulate neuroinflammation.
\end{abstract}

Keywords: microglia, phosphatidylinositol 3-kinase, mammalian target of rapamycin, glycogen synthase kinase-3, Akt, prostaglandins

\section{Findings}

Inflammation has been recognized not only as a mere bystander in neurodegenerative diseases but also as a factor driving disease progression. Microglia, the innate phagocytic cells of the central nervous system (CNS), constantly survey their microenvironment. Activated

\footnotetext{
* Correspondence: bernd.fiebich@uniklinik-freiburg.de

'Department of Psychiatry and Psychotherapy, University of Freiburg Medical School, Hauptstr. 5, D-79104 Freiburg, Germany

Full list of author information is available at the end of the article
}

microglia secrete inflammatory mediators, which could contribute to neuronal damage. Different groups have demonstrated that the inflammatory cyclooxygenase-2 (COX-2), inducible nitric oxide synthase (iNOS) and cytokines, such as interleukin (IL)-1 $\beta$, IL- 6 and tumor necrosis factor (TNF)- $\alpha$, are associated with neurodegenerative diseases [1]. Thus, reduction of microglia activation is an important target in the treatment of neurodegenerative diseases. Therefore, much effort has been made to identify intracellular pathways that are

\section{(Ciomed Central}


responsible for the expression of these pro-inflammatory mediators. However, many intracellular pathways which are involved in the production of inflammatory mediators by microglia are not well characterized. In particular, the role of the phosphatidylinositol 3-kinase (PI3K) signal cascade in mediating neuroinflammatory processes is poorly studied.

The PI3K pathway can be activated by different stimuli including LPS via the toll-like receptor $4 / C D 14$ receptor complex in microglia. After activation, PI3K phosphorylates phosphatidylinositol 4,5-bisphosphate to generate phosphatidylinositol-3,4,5-trisphosphate. The latter molecule binds to the pleckstrin homology domain of one of the Akt (also known as protein kinase B) isoforms and facilitates the phosphorylation of Akt1, Akt2 or Akt3 at $\mathrm{Thr}^{308 / 309 / 305}$ and $\mathrm{Ser}^{273 / 474 / 472}$, respectively, by the phosphatidylinositol-dependent kinases 1 and 2 [2]. The phosphorylation on the respective residues of Akt leads to further catalytic activity changes of downstream targets, such as glycogen synthase kinase-3 (GSK-3) and mammalian target of rapamycin (mTOR) $[3,4]$.

Recently, we and others have demonstrated that PI3K might play an important role in inflammation and microglia activation. In particular, we have demonstrated that COX-2 is up-regulated and microsomal prostaglandin E synthase-1 (mPGES-1) is down-regulated by the PI3K inhibitor LY294002 [5]. However, downstream pathways of PI3K might also be important. In order to investigate this issue, we utilized a pharmacological approach to further investigate the role of PI3K and downstream pathways in the expression of COX-2 and mPGES- 1 by activated microglia.

Primary microglial cell cultures were established from cerebral cortices of one-day neonatal Wistar rats [6] as described in detail in our recent study [5]. The purity of the microglial culture obtained in our experiments was $>98 \%$ as determined by immunofluorescence and cytochemical analysis according to the method developed by Gebicke-Haerter et al. (1989) [7]. To investigate the effect of the inhibition of downstream pathways of PI3K, the following compounds were used: the PI3K inhibitors LY294002 and PI828, as well as LY303511, the inactive analogue of LY294002 (all from Tocris, Ellisville, MO, or Calbiochem, Bad Soden, Germany); Akt inhibitor X and mTOR inhibitor rapamycin (both from Calbiochem, Bad Soden, Germany); the dual PI3K/mTOR inhibitor NVP-BEZ235 (Axon Medchem BV, Groningen, The Netherlands); the GSK-3 inhibitor SB216763 (Tocris, Ellisville, MO); LPS (from Salmonella typhimurium, Sigma-Aldrich, Taufkirchen, Germany). Stock solutions (5-10 mM) were prepared in dimethyl sulfoxide (DMSO) and stored at $-20^{\circ} \mathrm{C}$. Further dilutions were carried out in
DMSO and final concentration of DMSO for all concentrations of the drugs in culture medium was $0.1 \%$. All compounds, used at the given concentrations, did not affect the viability of the cells as observed through the MTT cell viability assay (data not shown).

To analyze COX-2 and mPGES-1 protein levels, cells were incubated with the respective inhibitors for $30 \mathrm{~min}$ followed by $48 \mathrm{~h}$ stimulation with LPS. In the analysis of phosphorylation of $\mathrm{p}-70 \mathrm{~S} 6 \mathrm{~K}$, a downstream target of mTOR, cells were incubated with the inhibitors for 30 min followed by $1 \mathrm{~h}$ stimulation with LPS. 30 to $50 \mu \mathrm{g}$ of protein from each sample was subjected to SDSPAGE on a $10-15 \%$ gel under reducing conditions. Primary antibodies were goat anti-COX-2 (M-19, Santa Cruz, Heidelberg, Germany) diluted 1:500 in Tris-buffered saline (TBS) containing 0.1\% Tween 20 (Merck, Darmstadt, Germany) and 1\% bovine serum albumin (BSA, Sigma-Aldrich), rabbit anti-mPGES-1 (Oxford Biomedical Research, 1:1000), rabbit anti-phosphop70S6K (Cell Signaling Technology, Beverly, MA, USA, 1:1000), rabbit anti-actin (Sigma, 1:5000). Proteins were detected with horseradish peroxidase (HRP)-coupled rabbit anti-goat IgG (Santa Cruz, 1:100,000) or HRPcoupled donkey anti-rabbit (GE Healthcare, Freiburg Germany, 1:25,000) using chemiluminescence (ECL) reagents (GE Healthcare).

To investigate the effect of Akt inhibitor X on cytosolic prostaglandin E synthase (cPGES) and mPGES-2, we performed real time PCR. Cells were pre-incubated with Akt X inhibitor at different concentrations $(0.1-5 \mu \mathrm{M})$ and LPS $(10 \mathrm{ng} / \mathrm{ml})$ was subsequently added for total 24 h. RNA preparation was done by using RNAspin mini RNA isolation kit (GE Healthcare) and for cDNA synthesis one microgram of total RNA was reverse transcribed using M-MLV reverse transcriptase and random hexamers (Promega, Mannheim, Germany). The synthesized cDNA was the template for the real-time polymerase chain reaction (PCR) amplification carried out by the CFX96 real-time PCR detection system (Bio-Rad Laboratories Inc.). Specific Probes and primers were designed by using Universal probe library (Roche). Reaction conditions were $5 \mathrm{~min}$ at $95^{\circ} \mathrm{C}$, followed by 40 cycles of $10 \mathrm{~s}$ at $95^{\circ} \mathrm{C}, 30 \mathrm{~s}$ at $60^{\circ} \mathrm{C}$, and $1 \mathrm{~s}$ at $72^{\circ} \mathrm{C}$ followed by $10 \mathrm{~s}$ at $40^{\circ} \mathrm{C}$. S12 served as an internal control for sample normalization and the comparative cycle threshold Ct method was used for data quantification as described previously [8]. The following primer sequences were used for mPGES-2: Forward 5'AGGAAGGTACCCATCCTGGT-3', Reverse 5'-GAGGAGTCATTGAGCTGTTGC-3'; cPGES: Forward 5'TGTCTAATTTTGACCGTTTCTCTG-3', Reverse 5'TCATCTGCTCCGTCTACTTCTG-3'; S12: Forward 5'GCGCTTAAATACCGTCATGC-3', Reverse: 5'GACGCCGAATCTTGAACG-3'. 
To determine $\mathrm{PGE}_{2}$ and $\mathrm{PGD}_{2}$ concentrations, cells were incubated with the respective inhibitors previously for $30 \mathrm{~min}$ followed by $48 \mathrm{~h}$ stimulation with LPS. Supernatants were harvested for the measurement of the levels of $\mathrm{PGD}_{2}$ (Cayman Chemicals, Ann Arbor, MI, USA) and $\mathrm{PGE}_{2}$ (AssayDesign, distributed by Biotrend, Köln, Germany). All measurements were performed according to the manufacturer's instructions. The standards were used in the interval of $39-2500 \mathrm{pg} / \mathrm{ml}$ (sensitivity of the assay was $36.2 \mathrm{pg} / \mathrm{ml}$ ) for both prostaglandins.

All experiments were carried out at least three times. Original data were converted into \% - values of LPS control and mean \pm S.E.M. were calculated. Values were compared using $t$-test (two groups) or one-way ANOVA with post-hoc Student-Newman-Keuls test (multiple comparisons). The level of statistical significance was set at a $\mathrm{p}$ value less than 0.05 .

We have recently demonstrated that LY294002, a PI3K inhibitor, reduces mPGES-1 and increases COX-2 expression, providing an interesting pattern of differential expression between these two enzymes [5]. To further investigate the contribution of pathways downstream of PI3K, we inhibited Akt with Akt inhibitor X. As shown in Figure 1, Akt inhibitor X slightly increased COX-2 and reduced mPGES-1 protein levels induced by LPS, although without reaching statistical difference (Figure 1A-B). On the other hand, the Akt inhibitor X increased significantly the production of $\mathrm{PGE}_{2}$ and $\mathrm{PGD}_{2}$ in LPS-activated microglia (Figure $1 \mathrm{C}$ ). To investigate whether the enhancement of $\mathrm{PGE}_{2}$ was due to an increase in the expression of other prostaglandin synthases, we investigated the effect of Akt inhibitor $\mathrm{X}$ on mPGES-2 and cPGES. Interestingly, we found mPGES-2 to be increased after $24 \mathrm{~h}$ stimulation with LPS, albeit cPGES levels remained unchanged. However, Akt inhibitor $\mathrm{X}$ did not affect the expression of both enzymes (Figure 1D).

Since the involvement of Akt in the enhanced production of prostanoids was suggested by using Akt inhibitor $\mathrm{X}$, we asked whether other downstream targets of PI3K/ Akt are affected. An important target of Akt is GSK-3. Different studies have demonstrated that Akt inactivates GSK-3 by phosphorylating a serine residue of this
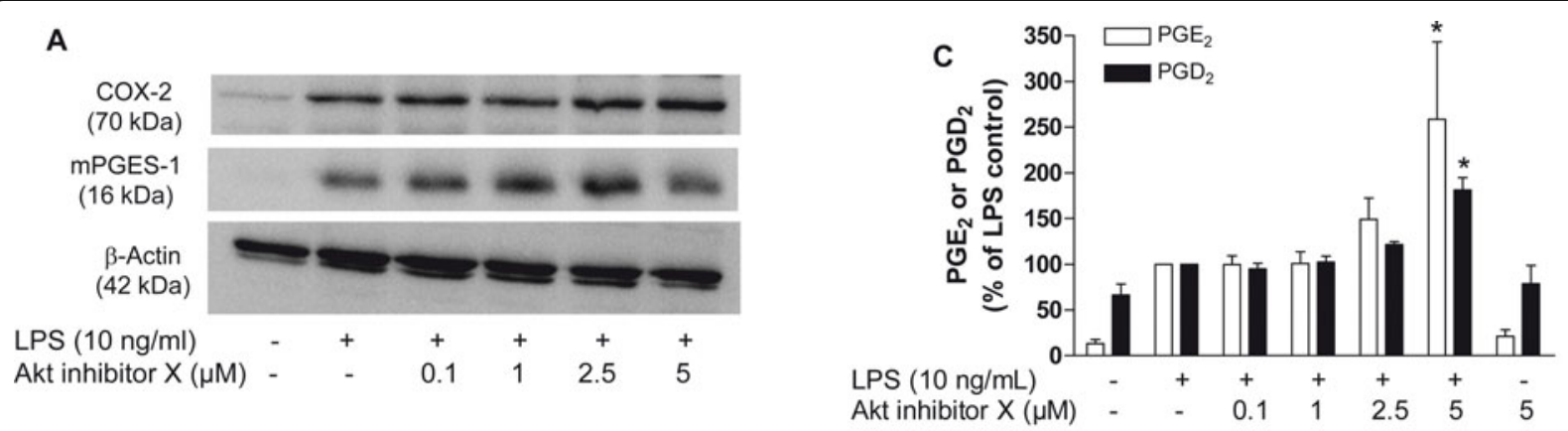

B
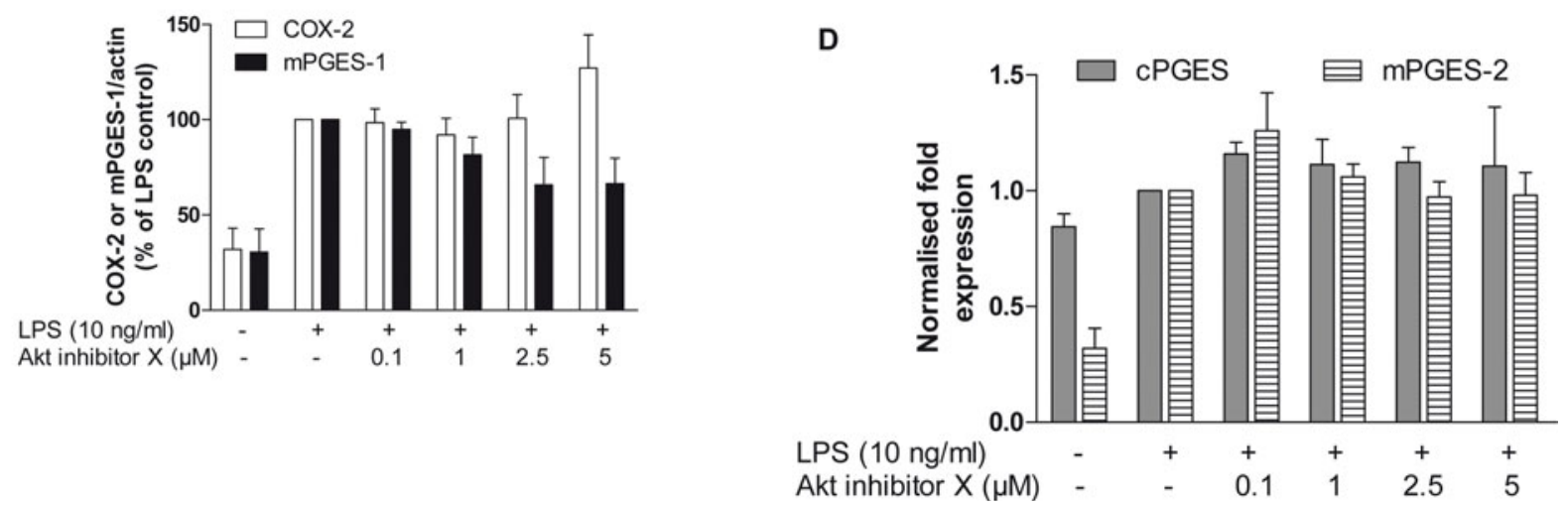

Figure 1 Effect of Akt inhibitor $X$ on COX-2 and mPGES-1 immunoreactivity and $\mathrm{PGE}_{2}$ and $\mathrm{PGD}_{2}$ release in primary LPS-stimulated rat microglia. A: Immunoblot analysis of protein levels of COX-2, mPGES-1 and $\beta$-actin in LPS-activated microglia treated with Akt inhibitor X (0.1-5 $\mu M)$. B: Quantitative densitometric analysis of COX-2 and mPGES-1 protein expression normalized to $\beta$-actin loading control. C: Effect of Akt inhibitor $X(0.1-5 \mu \mathrm{M})$ on $\mathrm{PGE}_{2}$ and $\mathrm{PGD}_{2}$ production after $48 \mathrm{~h}$ of LPS stimulation in rat primary microglia. $\mathbf{D}$ : Effect of Akt inhibitor $\mathrm{X}$ on mPGES2 and CPGES expression in primary LPS-stimulated rat microglia. *P $<0.05$ with respect to LPS control. 
enzyme [9]. This means that blockade of PI3K/Akt keeps GSK-3 in the active state, which might lead to increased COX-2 levels. Thus, inhibition of GSK-3 could potentially decrease COX-2 expression. Our data showed that the higher dose of the GSK-3 inhibitor SB216763 $(10 \mu \mathrm{M})$, significantly decreased COX-2 and mPGES-1 immunoreactivity induced by LPS in primary rat microglia (Figure 2A-B).

Thereafter, we asked whether mTOR inhibition alters LPS-induced COX-2 and mPGES-1 protein synthesis. Interestingly, rapamycin, a well-known mTOR inhibitor, increased both COX-2 and mPGES-1 immunoreactivity (Figure 3A-B). The production of $\mathrm{PGE}_{2}$ and $\mathrm{PGD}_{2}$ was also strongly enhanced by rapamycin in LPS-stimulated microglia (Figure 3C), but not in non-stimulated cells (Figure 3D).

Considering the possible involvement of PI3K and mTOR in the regulation of mPGES- 1 and COX-2 in

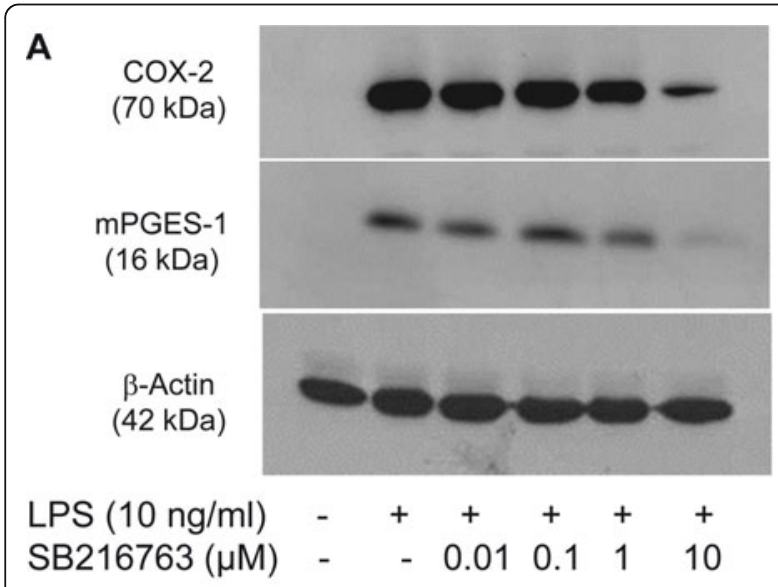

B

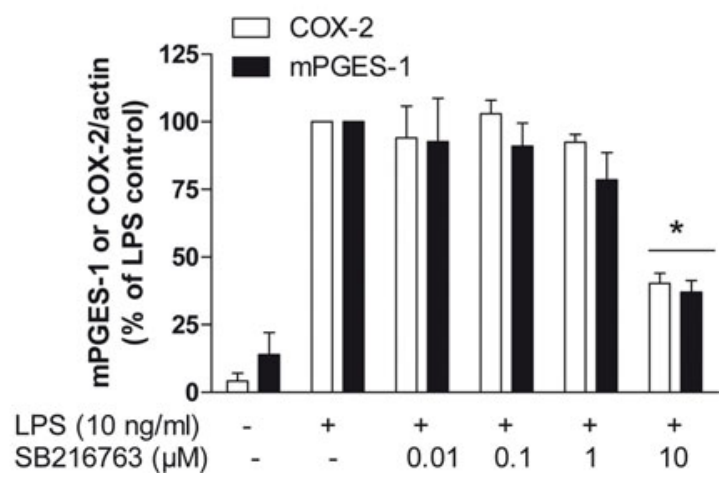

Figure 2 Effect of SB216763, a GSK-3 inhibitor, on mPGES-1 and COX-2 immunoreactivity in primary LPS-stimulated rat microglia. A: Immunoblot analysis of protein levels of COX-2, mPGES-1 and $\beta$-actin in LPS-activated microglia treated with SB216763 (0.01-10 $\mu \mathrm{M})$. B: Quantitative densitometric analysis of mPGES-1 and COX-2 protein expression normalized to $\beta$-actin loading control. ${ }^{*} \mathrm{P}<0.05$ with respect to LPS control. microglia, we investigated the effect of NVP-BEZ235, a dual PI3K/mTOR inhibitor. NVP-BEZ235 strongly enhanced COX-2, but reduced mPGES-1 immunoreactivity in activated microglia (Figure 4A-B). The synthesis of $\mathrm{PGE}_{2}$ and $\mathrm{PGD}_{2}$ was also strongly enhanced (Figure 4C). We further investigated whether NVP-BEZ235 increases COX-2 and mPGES-1, as well as prostaglandin synthesis, in absence of LPS. As shown in Figure 4D-E, there was a trend to enhancement of COX-2 and mPGES-1 expression. However, $\mathrm{PGE}_{2}$ synthesis was increased in non-stimulated microglia (Figure 4F).

As expected, the PI3K inhibitors, LY294002 and PI828, as well as rapamycin and NVP-BEZ235, reduced the phosphorylation of p-70S6K, an indirect marker of mTOR activation (Figure 4G). The PI3K inactive analogue LY303511 did not change this parameter.

In the present study, we investigated the role of Akt and downstream pathways in microglia activation with special emphasis on the arachidonic acid cascade. We provide new evidence that inhibition of these pathways significantly influence microglia activation.

We have previously demonstrated that PI3K inhibition reduced mPGES-1 and increased COX-2 synthesis in activated microglia. Here we further investigated whether inhibition of Akt and downstream pathways contributes to the regulation of COX-2 and mPGES-1 in LPS-stimulated primary microglia. Although there was a trend towards an increased expression of COX-2 and a reduction in the expression of $\mathrm{mPGES}-1$ with the exposure of the cells to Akt inhibitor X, no statistical difference was observed (Figure 1A-B). We have also observed that Akt inhibitor $\mathrm{X}$ did not change the expression of mPGES-2 and cPGES. However, as can be observed in Figure 1D, mPGES-2 is enhanced after $24 \mathrm{~h}$ incubation of cells with LPS. Although most studies indicate that mPGES-2 is a constitutive enzyme that is not inducible, the finding of the present study is interesting and is in accordance with some data that indicates that mPGES-2 can be enhanced in some conditions [10-12]. For example, Chaudhry et al. [10] have shown that mPGES-2 is expressed in activated, but not resting microglia of humans.

The highest dose of Akt inhibitor X significantly increased $\mathrm{PGE}_{2}$ and $\mathrm{PGD}_{2}$, indicating that a little enhancement in COX-2 expression is sufficient to increase prostanoid production (Figure 1C). The enhancement of $\mathrm{PGE}_{2}$ and $\mathrm{PGD}_{2}$ could be due to the slight, though not significant, enhancement of COX-2 expression. However, there are some other possibilities that could contribute to the enhancement of $\mathrm{PGE}_{2}$ and $\mathrm{PGD}_{2}$. Akt inhibition could enhance the expression and activity of $\mathrm{PLA}_{2}$ and COX-1, therefore enhancing the available arachidonic acid and $\mathrm{PGH}_{2}$, which are substrates for the synthesis of prostanoids. Moreover, it is 


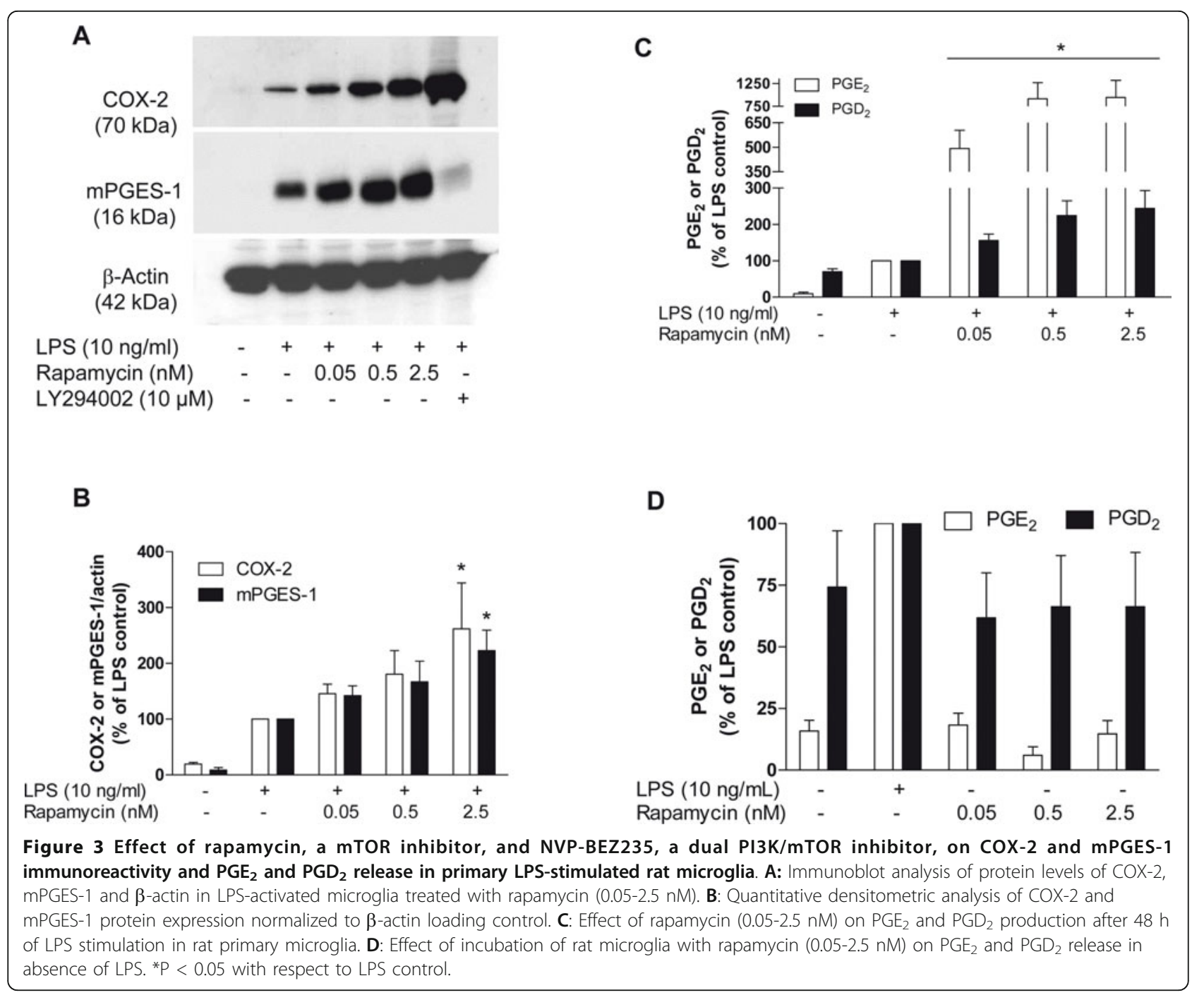

possible that Akt inhibitor $\mathrm{X}$ could also enhance the enzymatic activity of the COX-2 and the PGE and PGD synthases.

We further investigated downstream pathways of Akt, such as GSK-3. As expected, a selective GSK-3 inhibitor reduced COX-2 and mPGES-1 immunoreactivity (Figure $2 \mathrm{~A}-\mathrm{B})$. Although SB216763 has an $\mathrm{IC}_{50}$ in the $\mathrm{nM}$ range, it needs to be emphasized that this determination was performed with the isolated human enzyme [13], a condition that differs from the studies that use whole cells. In fact, different pharmacological effects have been demonstrated in macrophages and microglia exposed to this higher concentration [14-18].

Similar to our results, Takada et al. [19] have shown that genetic deletion of GSK-3 $\beta$ reduces the activation of NF- $\kappa \mathrm{B}$ and COX-2 expression induced by TNF $\alpha$-stimulated mouse embryonic fibroblasts. On the other hand, previous work has shown that GSK-3 $\beta$ inhibition results in COX-2 expression [20-22]. Moreover,
Yuskaitis and Jope [18] showed that GSK-3 inhibition by the use of different GSK-3 inhibitors is not important for COX-2 expression in LPS-stimulated BV-2 microglia. This difference between the studies can be due to the time of stimulation, the compounds used and the cell types used, since we used primary rat microglia and the authors of this independent study used the BV-2 cell line. Although SB216763 has been shown to be selective for GSK-3 over many other kinases [13], there is still the possibility that other targets other than GSK-3 are involved in COX-2 regulation. Some studies have addressed the role of GSK-3 in COX-2 regulation, but no data have been published concerning the role of GSK-3 in mPGES-1 expression.

Besides GSK-3, another important target of Akt is mTOR. Interestingly, here we demonstrate that COX-2 and mPGES-1 immunoreactivity, as well as $\mathrm{PGE}_{2}$ and $\mathrm{PGD}_{2}$, were drastically increased by rapamycin, a selective inhibitor of mTOR, even at very low doses (Figure 


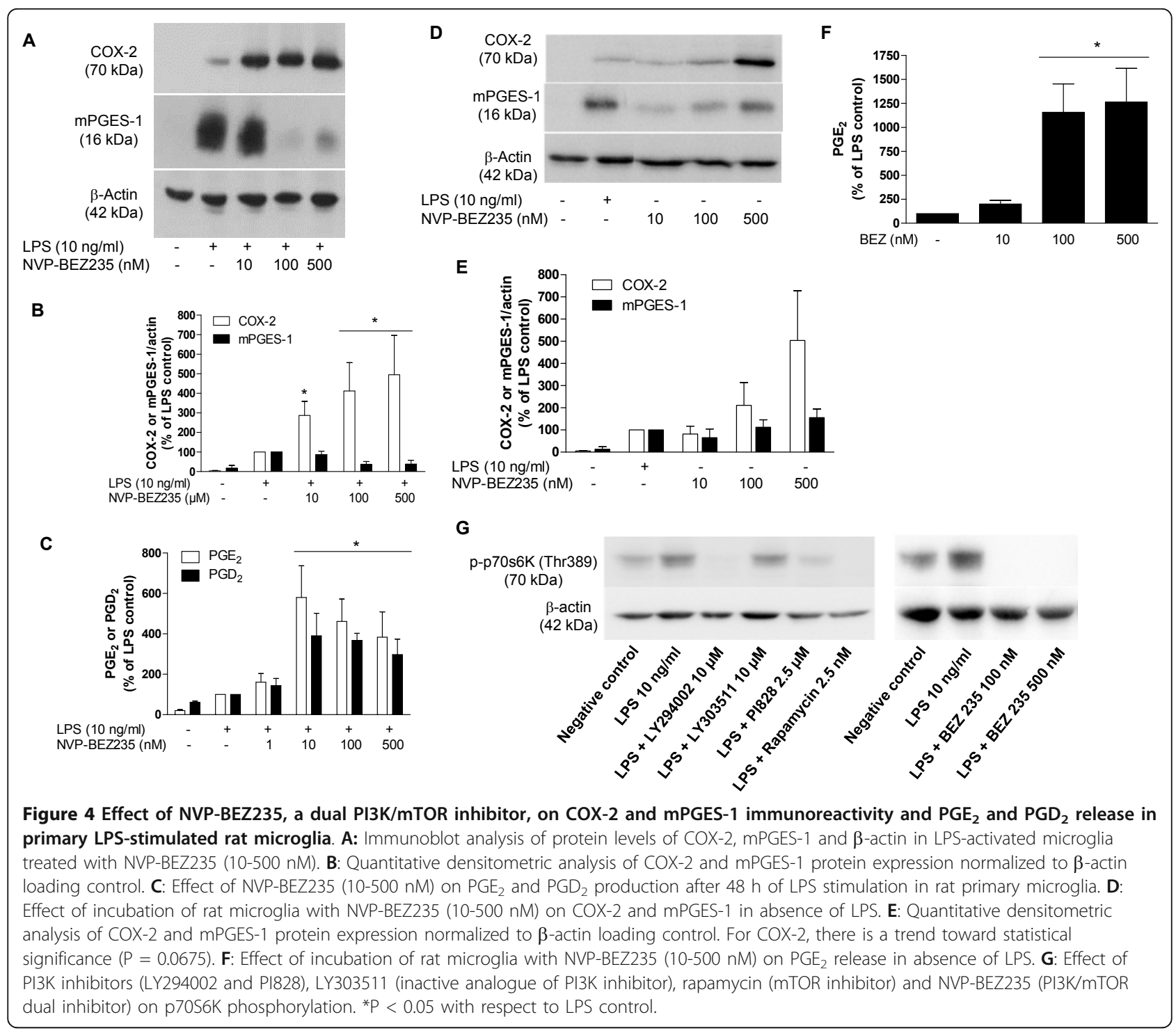

3A-C). To our knowledge, this is the first demonstration that rapamycin increases the production of prostaglandins. It has been shown that mTOR inhibitors reduced NOS activity and iNOS expression induced by hypoxia and a mixture of cytokines in BV-2 cells and primary rat microglia, respectively [23,24]. However, Dello Russo et al. [23] did not find any increment in the COX-2 expression with the association of a mixture of cytokines and RAD001, another mTOR inhibitor. This discrepancy between the studies might be due to the type of stimulus and a difference in the chemical structure of the drugs used.

Although rapamycin enhanced COX-2 and mPGES-1 protein levels, NVP-BEZ235, the dual PI3K/mTOR inhibitor, strongly increased COX-2, but reduced mPGES-1 immunoreactivity induced by LPS in microglia (Figure 4A-B). In fact, we have already previously demonstrated that the regulation of these two enzymes is not strictly coupled [5]. Interestingly, $\mathrm{PGE}_{2}$ and $\mathrm{PGD}_{2}$ were enhanced in LPS-stimulated. One hypothesis to explain the enhancement of $\mathrm{PGE}_{2}$ with $\mathrm{mPGES}-1$ reduction might be that the remaining $\mathrm{mPGES}-1$ expression, together with cPGES and mPGES-2, which are expressed in microglia, would be enough to produce the high levels of $\mathrm{PGE}_{2}$. Moreover, the activity of the enzymes could also be increased by the compound.

There are other studies demonstrating that $\mathrm{PGE}_{2}$ could be enhanced even with $\mathrm{mPGES}-1$ reduction. LY294002, a PI3K inhibitor, reduce mPGES-1 and enhance COX-2 expression, although the production of $\mathrm{PGE}_{2}$ and $\mathrm{PGD}_{2}$ is enhanced or not affected in LPS-stimulated microglia [5]. Moreover, it has been demonstrated that silencing mPGES-1 does not affect $\mathrm{PGE}_{2}$ production in IL-1 $\beta$ or TNF $\alpha$-stimulated gingival 
fibroblasts [25]. Similarly, this study demonstrated that MK-886, an inhibitor of 5-lipoxygenase-activating protein, reduced mPGES-1 and increased COX-2 expression, but did not affect $\mathrm{PGE}_{2}$ synthesis. In fact, different data suggest that $\mathrm{COX}-2$ might the rate-limiting enzyme in the synthesis of $\mathrm{PGE}_{2}$ [26-28].

Importantly, NVP-BEZ235 per se is able to increase $\mathrm{PGE}_{2}$ in non-stimulated cells. This result suggests that the dual PI3K/mTOR inhibitor might differently regulate the expression of inflammatory mediators in different conditions, since it reduces mPGES-1 induced by LPS and also upregulates this enzyme in non-stimulated conditions. That the increase in $\mathrm{PGE}_{2}$ mediated by NVP-BEZ235 is higher in non-stimulated in comparison to stimulated microglia is probably due to the fact that mPGES-1 is reduced in activated cells and increased in non-activated cells. Taken together, we provide evidence that the blockade of mTOR and/or PI3K/Akt enhances prostanoid production by microglia.

The results with rapamycin and NVP-BEZ235 represent important findings, since rapamycin is a commercially available drug used to prevent rejection of transplanted kidney and NVP-BEZ235 was recently identified [29] and is an orally bioavailable drug which is currently studied in clinical trials for advanced solid tumor patients. To date, only a few studies have demonstrated the effects of NVP-BEZ235 in non-tumor cells. Here we demonstrate that microglia may also be affected by a double PI3K/mTOR inhibition by modifying the production of inflammatory mediators in neuroinflammatory conditions. Considering that the arachidonic acid cascade products might have important roles in resolution and/or progression of neuroinflammation, the effects of these compounds should be investigated in vivo.

In conclusion, we provide novel evidence that Akt, GSK-3 and mTOR are important intracellular regulators of microglia activation. Our data provide significant information regarding the regulation of $\mathrm{COX}-2$ and mPGES-1 via the Akt/mTOR and Akt/GSK-3 pathways, and suggest that interfering with these signalling cascades using pharmacological inhibitors could modulate the activation state of microglial cells during neuroinflammation.

\section{Abbreviations used \\ Akt: protein kinase B; COX: cyclooxygenase; CPGES: cytosolic prostaglandin E synthase; GSK-3: glycogen synthase kinase-3; iNOS: inducible nitric oxide synthase; IL: interleukin; LPS: lipopolysaccharide; mPGES: microsomal prostaglandin E synthase; mTOR: mammalian target of rapamycin PG: prostaglandin; PI3K: phosphatidylinositol 3-kinase; TNF: tumor necrosis factor.}

\section{Acknowledgements}

The skilful technical assistance of Ulrike Götzinger-Berger and Brigitte Günter is greatly acknowledged. Antonio Carlos Pinheiro de Oliveira was supported by the CAPES Foundation (Brasilia/Brazil). Lena Wendeburg received a fellowship from Vivacell (Germany). Johannes CM Schlachetzki was supported by the ELAN program of the University Hospital Erlangen, Germany.

\section{Author details}

${ }^{1}$ Department of Psychiatry and Psychotherapy, University of Freiburg Medical School, Hauptstr. 5, D-79104 Freiburg, Germany. ${ }^{2}$ Department of Pharmacology, Universidade Federal de Minas Gerais, Av. Antonio Carlos 6627, 31270-901, Belo Horizonte, Brazil. ${ }^{3}$ Department of Neuroscience, University of Florida, Gainesville, FL 32610, USA. ${ }^{4}$ Department of Molecular Neurology, University of Erlangen, Erlangen, Germany. ${ }^{5}$ VivaCell Biotechnology GmbH, Ferdinand-Porsche-Str. 5, D-79211, Denzlingen, Germany.

\section{Authors' contributions}

$A C P d O, E C-J$, and BLF participated in research design. The experiments were performed by ACPdO, LW, JL and HSB. Data were analysed by ACPdO, EC-J, JCMS and HSB. ACPdO, EC-J, JCMS, BLF, and KB wrote or contributed to the writing of the manuscript. In addition, ACPdO, KB, EC-J, JCMS, and BLF reviewed the data and discussed the manuscript. All authors have read and approved the final version of the manuscript.

\section{Competing interests}

The authors declare that they have no competing interests.

Received: 13 July 2011 Accepted: 3 January 2012

Published: 3 January 2012

\section{References}

1. LiU B, Hong JS: Role of microglia in inflammation-mediated neurodegenerative diseases: mechanisms and strategies for therapeutic intervention. J Pharmacol Exp Ther 2003, 304:1-7.

2. Laine J, Kunstle G, Obata T, Noguchi M: Differential regulation of Akt kinase isoforms by the members of the TCL1 oncogene family. J Biol Chem 2002, 277:3743-3751.

3. Cantley LC: The phosphoinositide 3-kinase pathway. Science 2002, 296:1655-1657.

4. Fukao T, Koyasu S: PI3K and negative regulation of TLR signaling. Trends Immunol 2003, 24:358-363.

5. de Oliveira AC, Candelario-Jalil E, Bhatia HS, Lieb K, Hull M, Fiebich BL: Regulation of prostaglandin E2 synthase expression in activated primary rat microglia: evidence for uncoupled regulation of mPGES-1 and COX-2. Glia 2008, 56:844-855.

6. Seregi A, Keller M, Jackisch R, Hertting G: Comparison of the prostanoid synthesizing capacity in homogenates from primary neuronal and astroglial cell cultures. Biochem Pharmacol 1984, 33:3315-3318.

7. Gebicke-Haerter PJ, Bauer J, Schobert A, Northoff H: Lipopolysaccharidefree conditions in primary astrocyte cultures allow growth and isolation of microglial cells. J Neurosci 1989, 9:183-194.

8. Livak KJ, Schmittgen TD: Analysis of relative gene expression data using real-time quantitative PCR and the 2(-Delta Delta $C(T)$ ) Method. Methods 2001, 25:402-408.

9. Jope RS, Yuskaitis CJ, Beurel E: Glycogen synthase kinase-3 (GSK3): inflammation, diseases, and therapeutics. Neurochem Res 2007, 32:577-595.

10. Chaudhry U, Zhuang H, Dore S: Microsomal prostaglandin E synthase-2: cellular distribution and expression in Alzheimer's disease. Exp Neurol 2010, 223:359-365.

11. Kubota K, Kubota T, Kamei D, Murakami M, Kudo I, Aso T, Morita I: Change in prostaglandin E synthases (PGESs) in microsomal PGES-1 knockout mice in a preterm delivery model. J Endocrinol 2005, 187:339-345.

12. Zhang J, Fujii S, Wu Z, Hashioka S, Tanaka Y, Shiratsuchi A, Nakanishi Y, Nakanishi H: Involvement of COX-1 and up-regulated prostaglandin E synthases in phosphatidylserine liposome-induced prostaglandin E2 production by microglia. J Neuroimmunol 2006, 172:112-120.

13. Coghlan MP, Culbert AA, Cross DA, Corcoran SL, Yates JW, Pearce NJ, Rausch OL, Murphy GJ, Carter PS, Roxbee Cox L, et al: Selective small molecule inhibitors of glycogen synthase kinase- 3 modulate glycogen metabolism and gene transcription. Chem Biol 2000, 7:793-803.

14. Ha SD, Ng D, Pelech SL, Kim SO: Critical role of the phosphatidylinositol 3-kinase/Akt/glycogen synthase kinase-3 signaling pathway in recovery 
from anthrax lethal toxin-induced cell cycle arrest and MEK cleavage in macrophages. J Biol Chem 2007, 282:36230-36239.

15. Park SH, Park-Min KH, Chen J, Hu X, Ivashkiv LB: Tumor necrosis factor induces GSK3 kinase-mediated cross-tolerance to endotoxin in macrophages. Nat Immunol 2011, 12:607-615.

16. Ren F, Duan Z, Cheng Q, Shen X, Gao F, Bai L, Liu J, Busuttil RW, KupiecWeglinski JW, Zhai Y: Inhibition of glycogen synthase kinase 3 beta ameliorates liver ischemia reperfusion injury by way of an interleukin10-mediated immune regulatory mechanism. Hepatology 2011, 54:687-696.

17. Suh HS, Choi S, Khattar P, Choi N, Lee SC: Histone deacetylase inhibitors suppress the expression of inflammatory and innate immune response genes in human microglia and astrocytes. I Neuroimmune Pharmacol 2010, 5:521-532.

18. Yuskaitis CJ, Jope RS: Glycogen synthase kinase-3 regulates microglial migration, inflammation, and inflammation-induced neurotoxicity. Cell Signal 2009, 21:264-273.

19. Takada Y, Fang X, Jamaluddin MS, Boyd DD, Aggarwal BB: Genetic deletion of glycogen synthase kinase-3beta abrogates activation of lkappaBalpha kinase, JNK, Akt, and p44/p42 MAPK but potentiates apoptosis induced by tumor necrosis factor. J Biol Chem 2004, 279:39541-39554.

20. Rao R, Hao CM, Breyer MD: Hypertonic stress activates glycogen synthase kinase 3 beta-mediated apoptosis of renal medullary interstitial cells, suppressing an NFkappaB-driven cyclooxygenase-2-dependent survival pathway. J Biol Chem 2004, 279:3949-3955.

21. Tang Q, Gonzales M, Inoue H, Bowden GT: Roles of Akt and glycogen synthase kinase 3beta in the ultraviolet B induction of cyclooxygenase-2 transcription in human keratinocytes. Cancer Res 2001, 61:4329-4332.

22. Thiel A, Heinonen M, Rintahaka J, Hallikainen T, Hemmes A, Dixon DA, Haglund C, Ristimaki A: Expression of cyclooxygenase-2 is regulated by glycogen synthase kinase-3beta in gastric cancer cells. J Biol Chem 2006, 281:4564-4569.

23. Dello Russo C, Lisi L, Tringali G, Navarra P: Involvement of mTOR kinase in cytokine-dependent microglial activation and cell proliferation. Biochem Pharmacol 2009, 78:1242-1251.

24. Lu DY, Liou HC, Tang CH, Fu WM: Hypoxia-induced iNOS expression in microglia is regulated by the PI3-kinase/Akt/mTOR signaling pathway and activation of hypoxia inducible factor-1alpha. Biochem Pharmacol 2006, 72:992-1000

25. Bage T, Modeer T, Kawakami T, Quezada HC, Yucel-Lindberg T: Regulation of prostaglandin E synthases: effects of siRNA-mediated inhibition of microsomal prostaglandin E synthase-1. Biochim Biophys Acta 2007, 1773:1589-1598.

26. Degousee N, Martindale J, Stefanski E, Cieslak M, Lindsay TF, Fish JE, Marsden PA, Thuerauf DJ, Glembotski CC, Rubin BB: MAP kinase kinase 6p38 MAP kinase signaling cascade regulates cyclooxygenase-2 expression in cardiac myocytes in vitro and in vivo. Circ Res 2003, 92:757-764.

27. Mancini A, Jovanovic DV, He QW, Di Battista JA: Site-specific proteolysis of cyclooxygenase-2: a putative step in inflammatory prostaglandin $E(2)$ biosynthesis. J Cell Biochem 2007, 101:425-441.

28. Sevigny MB, Li CF, Alas M, Hughes-Fulford M: Glycosylation regulates turnover of cyclooxygenase-2. FEBS Lett 2006, 580:6533-6536.

29. Maira SM, Stauffer F, Brueggen J, Furet P, Schnell C, Fritsch C, Brachmann S, Chene P, De Pover A, Schoemaker K, et al: Identification and characterization of NVP-BEZ235, a new orally available dual phosphatidylinositol 3-kinase/mammalian target of rapamycin inhibitor with potent in vivo antitumor activity. Mol Cancer Ther 2008, 7:1851-1863.

doi:10.1186/1742-2094-9-2

Cite this article as: de Oliveira et al: Pharmacological inhibition of Akt and downstream pathways modulates the expression of COX-2 and mPGES-1 in activated microglia. Journal of Neuroinflammation 2012 9:2.

\section{Submit your next manuscript to BioMed Central and take full advantage of:}

- Convenient online submission

- Thorough peer review

- No space constraints or color figure charges

- Immediate publication on acceptance

- Inclusion in PublMed, CAS, Scopus and Google Scholar

- Research which is freely available for redistribution 